\title{
Enfermedad celíaca. Una mirada actual
}

\author{
M. Araya • K. Bascuñán
}

\section{ABSTRACT \\ Catching up on celiac disease}

Knowledge about celiac disease continues to grow and amaze those who investigate, seek and treat this condition. Gone are the days when it was considered just a rare child's digestive disease. It is now recognized as a highly prevalent autoimmune condition that affects children and adults with digestive and extra-digestive symptoms of diverse intensity, disorder that may be either mono, oligo or asymptomatic from a digestive point of view. Today, it is an underdiagnosed condition, not actively considered, and often mistakes are made regarding its diagnosis, treatment and gluten-free diet monitoring. This article reviews the current definition of the disease, clinical presentations, potential patients, how to search for the disease, how the diagnosis is made and characteristics of the treatment and monitoring of celiac patients, all based on internationally agreed standards, and emphasizing those aspects that have proven to be useful in other countries regarding the management of the disease.

(Key words: Celiac disease, clinical, active search, gluten-free diet).

Rev Chil Pediatr 2014; 85 (6): 658-665

\section{RESUMEN}

El conocimiento acerca de la enfermedad celíaca continúa creciendo y sorprendiendo a aquellos que la investigan, la buscan y la tratan. Lejos están los tiempos en que se la consideraba una patología digestiva del niño, poco frecuente. Actualmente se la reconoce como una condición autoinmune altamente prevalente, que afecta a niños y adultos, con manifestaciones digestivas y extra digestivas de muy diversa intensidad, pudiendo ser mono, oligo o incluso asintomática desde el punto de vista digestivo. Hoy en día está sub-diagnosticada, no se la busca activamente, se incurren en errores tanto al diagnosticarla como en la indicación del tratamiento y seguimiento de la dieta sin gluten. En este artículo revisamos la definición actual de la enfermedad, las presentaciones clínicas que se le conocen, en quiénes y cómo se debe buscar, como se hace el diagnóstico, y en qué consiste el tratamiento y seguimiento del paciente celíaco, basándonos en los criterios internacionales actualmente consensuados, y poniendo énfasis en aquellos aspectos que han demostrado ser útiles en otros paísespara mejorar el manejo de la enfermedad.

(Palabras clave: Enfermedad celíaca, clínica, búsqueda activa, dieta sin gluten).

Rev Chil Pediatr 2014; 85 (6): 658-665

Recibido el 14 de noviembre de 2013, aceptado para publicación el 10 de octubre de 2014

Magdalena Araya $(\varangle)$

Instituto de Nutrición y Tecnología de los Alimentos (INTA), Universidad de Chile.

E-mail: maraya@inta.uchile.cl

Karla Bascuñán

Departamento de Nutrición, Facultad de Medicina, Universidad de Chile. 


\section{Introducción}

El conocimiento acerca de la enfermedad celíaca (EC) continúa creciendo y sorprendiendo a aquellos que la investigan, la buscan y la tratan. Lejos están los tiempos en que se la consideraba una patología digestiva del niño, poco frecuente. Actualmente se la reconoce como una de las condiciones autoinmunes más altamente prevalente, que afecta a niños $\mathrm{y}$ adultos de todo el mundo ${ }^{1}$, con manifestaciones digestivas y extra digestivas de muy diversa intensidad, pudiendo ser mono, oligo o incluso asintomática. La EC deteriora en forma considerable la calidad de vida de los afectados, frecuentemente tiene manifestaciones autoinmunes de altos costos de tratamiento y se le ha reportado una mortalidad más elevada que el resto de la población ${ }^{2,3}$. Su tratamiento -que obliga una dieta especial, sin gluten, estricta y permanente, y que en general no incluye fármacos- es de alto costo económico $\mathrm{y}$ social. Tratándose de una condición que cada día se diagnostica con mayor frecuencia, es importante que los profesionales de la salud que atendemos a la población de celíacos del país nos pongamos al día en el tema; eso es lo que nos motiva a revisar en este artículo aquellos aspectos considerados cruciales para mejorar el manejo médico-nutricional de esta condición. Dado los importantes cambios actualmente en curso derivados del mejor conocimiento sobre esta condición, la opinión generalizada de los especialistas es seguir rigurosamente los criterios de la Sociedad Europea de Gastroenterología Pediátrica y Nutrición (ESPGHAN), que reúne a un enorme grupo de expertos quienes se reúnen periódicamente y reevalúan el estado del arte en el conocimiento de la EC, estableciendo los criterios de cómo manejarla. En este artículo seguiremos sus conceptualizaciones, focalizándonos en aquellos puntos aún poco claros y en los que Guías de otros grupos de especialistas pueden tener algunas diferencias ${ }^{4}$.

\section{¿Ha variado la definición de enfermedad celíaca?}

Desde que se describió la enfermedad en 1950, rápidamente se hizo evidente que sus manifestaciones clínicas eran a tal punto variables que no era conveniente definirla ni basar los criterios diagnósticos en la clínica de la enfermedad. En 1969 se logró el consenso internacional y se estableció que la enfermedad sería definida y manejada por los criterios que estableciera la Sociedad Europea de Gastroenterología Pediátrica y Nutrición (ESPGHAN), criterios que se han re evaluado periódicamente y hasta hoy son establecidos por el mismo grupo de especialistas 5 . A lo largo del tiempo se han incorporado otros grupos de opinión experta, por ejemplo el colegio Americano de Gastroenterólogos (ACG) ${ }^{4}$. Dado que no existe un modelo animal que permita estudiar esta patología en forma experimental, el estado del arte se fundamenta principalmente en series de pacientes descritos en todo el mundo ${ }^{6,7}$; hoy en día la biología celular y molecular contribuyen a entender aspectos específicos de la etiopatogenia y genética de la $\mathrm{EC}^{8}$, aunque hay que mantener presente que muchos de los conocimientos en estas áreas se basan en experimentación en modelos que constituyen un "proxi" y no la enfermedad propiamente tal.

La definición propuesta en la última actualización hecha por ESPGHAN en $2012^{5}$ dice que la EC es una condición que va más allá de sólo una enteropatía inducida por gluten, que era la definición aceptada hasta hace poco tiempo; es un desorden sistémico mediado inmunológicamente, gatillado por el gluten y prolaminas relacionadas, en individuos genéticamente susceptibles, y caracterizada por la presencia de una combinación variable de: 1) manifestaciones clínicas gluten-dependientes; 2) anticuerpos específicos de EC [antitTG2 (tTG) y anti-endomisio (EMA); 3) perfil genético de riesgo (haplotipos HLA DQ2 y DQ8) y 4) enteropatía (diferentes grados de infiltrado linfoplasmocitario y aplanamiento vellositario en la biopsia duodenal-yeyunal).

\section{Las presentaciones clínicas}

Las manifestaciones clínicas son diversas, desde pacientes oligo, mono o aparentemente asintomáticos hasta el cuadro clásico con síndrome de malabsorción asociado a desnutri- 
Tabla 1. Sintomatología descrita en la enfermedad celíaca y considerada sugerente de esta condición

\begin{tabular}{|lll|}
\hline Grupo etario & Manifestación digestiva & Manifestación extra digestiva \\
Lactante-Pre escolar & Diarrea crónica & Compromiso de estado general malnutrición \\
& Inapetencia & Hipotrofia muscular \\
& Vómitos & Retraso pondo-estatural \\
Dolor abdominal recurrente & Irritabilidad \\
& Distensión abdominal & Hipoferremia/anemia ferropriva \\
& Apatía/introversión/tristeza \\
\hline Escolar-Adolescente & Frecuentemente asintomático & Talla baja \\
& Constipación/diarrea crónica & Retraso puberal/retraso de la menarquia \\
& Dolor abdominal & Irregularidades menstruales/amenorrea \\
& Hábito intestinal irregular & Aftas orales \\
Distensión abdominal & Hipoplasia del esmalte \\
& Debilidad/hipotrofia muscular \\
& Artralgias/artritis/osteopenia \\
& Anemia ferropriva \\
\hline
\end{tabular}

ción (tabla 1). Las últimas Guías de ESPGHAN ponen énfasis en que la sintomatología debe responder al retiro del gluten de la dieta y distingue actualmente 5 presentaciones:

1) Con sintomas y signos gastrointestinales (diarrea crónica, dolor abdominal, distensión: abdominal, estreñimiento, etc.). Representa la forma "típica" o "clásica", con síndrome de malabsorción intestinal, aplanamiento importante (o total) de las vellosidades, y aparece más frecuentemente durante la infancia.

2) Con sintomas y signos extra intestinales. Pueden predominar manifestaciones que son consecuencia del síndrome de malabsorción subclínico (anemia por deficiencia de hierro que reaparece o no responde a tratamiento habitual, osteopenia u osteoporosis en edades tempranas, talla baja). Puede incluir procesos autoinmunes diversos (tiroiditis, hepatitis, nefropatía), infertilidad y alteraciones neuropsiquiátricas.

3) Silente: Se define por la presencia de anticuerpos específicos (EMA y/o tTG), marcadores HLA (DQ2/DQ8), y biopsia de intestino delgado compatible (grados 2 a 4 en la escala de Marsh) ${ }^{9}$, en ausencia de manifestaciones clínicas que sugieran o apoyen el diagnóstico de EC. Actualmente, estos casos se descubren frecuentemente por "búsqueda activa", al estudiar individuos que pertenecen a grupos de riesgo y a familiares directos de pacientes ya diagnosticados.

4) Latente: Se define por la presencia de marcadores genéticos compatibles (HLA DQ2/ DQ8) en ausencia de enteropatía, o bien son pacientes con diagnóstico de EC en algún momento de su vida, que respondieron clínica e histológicamente a la dieta sin gluten, y hoy permanecen asintomáticos, con anticuerpos negativos e histología del intestino delgado normal en circunstancias que su dieta contiene gluten en las cantidades habituales.

5) Potencial: Se define por presencia de anticuerpos específicos y marcadores genéticos HLA compatibles, sin manifestaciones clínicas ni anormalidades histológicas en biopsias duodenales.

Recientemente se han comenzado a reconocer cuadros aledaños a la EC, como son la sensibilidad al gluten y la alergia al trigo. Al respecto, se entiende por sensibilidad al gluten a un grupo de reacciones adversas asociadas a la ingesta de gluten, en las cuales el estudio del paciente no demuestra la presencia de fenómenos alérgicos o autoinmunes. Estos pacientes se caracterizan por presentar marcadores serológicos negativos (excepto IgG antigliadina, que puede ser positivo) y biopsias de duodeno normales ${ }^{10}$, con sintomatología sugerente de EC. Aunque actualmente se desconoce su patogenia y su relación con EC, la evidencia disponible sugiere una desregulación inmune, con disminución de la actividad de linfocitos $\mathrm{T}$ reguladores y el aumento de marcadores de inmunidad innata ${ }^{10}$. La Alergia al trigo, en cambio, se trata de una reacción adversa de 
origen inmune, gatillada por las proteínas del trigo, en ausencia de fenómenos de autoinmunidad; actualmente, el mecanismo subyacente está demostrado para reacciones de tipo IgE. El alérgeno puede entrar en contacto con el individuo por vía cutánea, digestiva o respiratoria. Puede manifestarse con síntomas cutáneos, gastrointestinales y respiratorios. A menudo el diagnóstico se basa en la presencia de un cuadro clínico caracterizado por una reacción rápida frente al contacto con trigo, asociado a la positividad en las pruebas cutáneas positivas para trigo y/o aumento de la IgE específica ${ }^{10}$. El tratamiento consiste en una dieta estricta de eliminación de alimentos que contengan trigo. La demostración de la presencia de IgE contra antígenos del trigo, en ausencia de síntomas asociados directamente a la exposición (sensibilización), no justifica la indicación de dieta de eliminación ni otras maniobras terapéuticas. En ambos casos, se recomienda que el diagnóstico y manejo sea realizado por un especialista, quien, cuando se trata de un paciente pediátrico, deberá explicar a los padres que la alergia en la edad infantil habitualmente es transitoria, mientras que la EC es crónica y requiere dieta sin gluten a lo largo de toda la vida. También les debe explicar que en los cuadros de alergia se debe evitar toda presencia del alérgeno, mientras que en la EC la dieta sin gluten implica un punto de corte, bajo el cual el paciente puede consumir el alimento sin correr riesgos.

\section{¿En quienes debemos buscar enfermedad celíaca?}

En la última década ha quedado claro que el número de pacientes celíacos conocidos es muy inferior al que está diagnosticado $^{11-13}$. En buena parte esto se debe a que, anteriormente, la enfermedad se buscaba solamente en personas que se sentían enfermas y consultaban al médico. El acceso a los anticuerpos EMA y tTG, y posteriormente su uso como herramienta de rastreo en grandes grupo de población, permitió establecer la existencia de patologías en
Tabla 2. Patologías en las que debe buscarse activamente la enfermedad celíaca por representar condiciones de riesgo

\begin{tabular}{|ll}
\hline Autoinmunes & No autoinmunes \\
Diabetes Mellitus tipo 1 & Familiar directo de celíaco conocido \\
Tiroiditis autoinmune & Síndrome de Down \\
Hepatitis autoinmune & Síndrome de Turner \\
Enfermedad de Addison & Síndrome de Williams \\
Síndrome de Sjôgren & $\begin{array}{l}\text { Déficit selectivo de IgA } \\
\text { Infertilidad }\end{array}$
\end{tabular}

las que la EC es mucho más frecuente que en la población general ${ }^{14,15}$. Esto llevó al concepto de "búsqueda activa", es decir, buscar la presencia de estos anticuerpos en todos aquellos individuos que contactan el sistema de salud, por diversas sintomatologías, y que tienen alguna de las patologías en las que se han descritos frecuencias de EC más altas que en la población general (tabla 2 ); en aquellos que resultan positivos se debe confirmar el diagnóstico mediante biopsia de intestino delgado. Este protocolo es el que ha llevado a establecer que la prevalencia de la EC es del orden del $0,5-1 \%$ en la mayoría de las poblaciones estudiadas, de diversas etnias y orígenes ${ }^{11}$. En Chile, la segunda Encuesta Nacional de Salud ${ }^{16}$ determinó que poco menos del 1\% de población tiene IgA-tTG positivo, o sea, es población llamada "susceptible". La frecuencia de la enfermedad propiamente tal se estima actualmente en $\sim 0,5 \%$ de la población chilena, en contraste a la prevalecía de 1:1.800 que se conocía anteriormente en base a los casos diagnosticados a propósito de su consulta al especialista ${ }^{17}$. Esta práctica de buscar activamente a los pacientes, sin esperar a que consulten, ha tenido dos consecuencias interesantes: los familiares de primer grado de celíacos, diagnosticados por búsqueda activa, representan alrededor de $10-15 \%$ de los casos actualmente diagnosticados; por otra parte, la proporción de casos diagnosticados ha aumentado considerablemente entre los adultos, con una disminución relativa de casos cuya sintomatología principal es diarrea durante la edad pediátrica ${ }^{18,19}$.

\section{Diagnóstico}

Ante la amplia variedad de presentaciones clínicas el cómo se diagnostican algunos casos ex- 
cepcionales de EC se ha convertido en tema de debate. Es por esto que ESPGHAN en 2012 revisó y puso al día los criterios para hacer el diagnóstico. Establecen que se debe hacer búsqueda activa en individuos con condiciones de riesgo, que incluye a los familiares directos (hermanos, padres, hijos) de un caso ya diagnosticado (tabla 2) y se debe evaluar a los pacientes que desarrollan sintomatología (digestiva y/o extra digestiva) sugerente de la enfermedad. El diagnóstico se basa en ${ }^{5,19}$ :

1. Evidencia clínica sugerente, a excepción de ser un caso evaluado por búsqueda activa por pertenecer a un grupo de riesgo.

2. Anticuerpos IgA-tTG2* y/o IgA-EMA positivos* e IgA sérica normal**

3. Biopsia de intestino delgado*** con daño moderado a grave de la mucosa (categorías de Marsh 2 o más).

*= hoy en día tTG2 es el auto antígeno más usado para estudios serológicos. Su medición es cuantitativa, mediante un test de ELISA, que mide la cantidad de anticuerpos presente en la sangre; su sensibilidad y especificidad están descritas en alrededor de 90\%. Los kit desarrollados en años recientes usan anticuerpos dirigidos a nuevos epítopes, distintos a los originalmente usados, y están resultando más sensibles que las versiones iniciales. El endomisio es la matriz extracelular donde se expresa la tTG2. La determinación de EMA es cualitativa, se mide por inmuno fluorescencia y demuestra la presencia o ausencia de anticuerpos. La sensibilidad descrita para IgA-EMA es entre 86 a $90 \%$ y la especificidad entre el 97 y $100 \%$. Cuando se hacen los dos anticuerpos, si son concordantes, su sensibilidad y especificidad son cercanas al $100 \%$.

$* *=$ la deficiencia de $\operatorname{IgA}$ es más frecuente entre los celíacos que en la población general. Junto con los anticuerpos IgA-EMA e IgAtTG2, en todos los casos se debe solicitar la medición de IgA sérica ${ }^{20}$. En los pacientes con deficiencia de IgA se deberá repetir la medición de los anticuerpos en sus versiones IgGtTG y/o IgG-EMA.

$* * *=$ la biopsia debe hacerse -siempre- antes de indicar la DLG. En los familiares que se estudian porque hay un celíaco conocido en la familia, debe asegurarse que tienen una ingesta habitual de gluten al momento de ser estudiados.

Es importante comentar el debate producido por una situación de excepción establecida por ESPGHAN ${ }^{5}$ : establecen que, en niños evaluados por un especialista, en presencia de clínica típica y florida, además de niveles superiores a diez veces el valor límite de la normalidad en tTG2, con EMA positivo y haplotipos de riesgo positivos (DQ2/DQ8), se puede prescindir de la biopsia intestinal para el diagnóstico. Es muy importante hacer énfasis en que el estudio serológico y la biopsia deben hacerse-siempre- antes de iniciar la dieta sin gluten.

\section{¿Qué hay de nuevo en el tratamiento de EC?}

La piedra angular del tratamiento es la eliminación del gluten de la dieta. La Dieta Libre de Gluten (DLG) evita el consumo de las proteínas de almacenaje presentes en el trigo, centeno y cebada y algunos híbridos de estos cereales tales como el kamut y el triticale, de consumo excepcional en nuestro país. Diversos estudios indican que estos granos contienen los epítopes en los cuales la deamidación es importante para la unión a DQ2 y el reconocimiento por las células $\mathrm{T}$, conduciendo al daño observado en la $\mathrm{EC}^{21,22}$. En la gran mayoría de los pacientes la DLG lleva a la resolución completa de la sintomatología y de las alteraciones histológicas propias de la enfermedad. El incumplimiento de la dieta es la causa principal de la falta de respuesta al tratamiento; estos pacientes deben diferenciarse de aquellos "refractarios", o sea que no responden a la DLG o bien dejan de responder a ella; dicho diagnóstico diferencial debe hacerlo un especialista y a nivel terciario. Por esto, la evaluación minuciosa, la orientación al momento del diagnóstico y el cuidado continuo a lo largo de la vida resultan cruciales ${ }^{23}$.

Hasta hoy la EC no puede ser prevenida, actualmente el gran estudio europeo PREVENTCD está evaluando si la modalidad de alimentación durante el primer año de vida puede prevenir o al menos retardar la aparición de la enfermedad. Un estudio reciente conclu- 
yó que el riesgo de desarrollar EC se redujo a la mitad cuando la introducción inicial del gluten ocurre cuando el lactante aún recibe lactancia materna, existiendo además una correlación inversa entre la duración de la lactancia materna y el riesgo de desarrolla $\mathrm{EC}^{24}$. La recomendación actual de la ESPGHAN es introducir el gluten por primera vez en la dieta, entre los $4 \mathrm{y}$ 7 meses de edad ${ }^{25}$. Este rango de edad aumentaría la probabilidad de que el lactante aun esté recibiendo leche materna, que haya una mayor eficacia de los mecanismos de inducción de tolerancia oral y una adecuada maduración de la integridad de la mucosa intestinal.

La inclusión de avena y almidón de trigo en la DLG es controversial. Estudios a corto y largo plazo en la última década, involucrando población adulta ${ }^{26-28}$ y pediátrica ${ }^{29,30}$, sugieren que la avena se puede incluir de forma segura en alimentos "libres de gluten". Más aún, Størsrud $^{31}$ encontró que el uso de avena en la DLG aumentó la ingesta de hierro, fibra dietética, tiamina y zinc en pacientes celíacos. Sin embargo, estos estudios han sido relativamente pequeños, pocos han sido doble ciego o aleatorizados y sólo uno ha tenido una duración superior a un año. Independiente de la discusión de si las prolaminas de la avena tienen efectos adversos para el celíaco, la recomendación actual es no incluir avena en la DLG, porque se han demostrado altos niveles de contaminación en las avenas comerciales, describiéndose desde 1,5 ppm hasta más de 400 ppm, haciendo las mediciones en un mismo envase ${ }^{32}$; por otro lado, aunque escasas, existen descripciones de pacientes que han demostrado ser sensibles a la avena ${ }^{33}$.

La inclusión de productos a base de almidón de trigo también es motivo de controversia. Los primeros estudios realizados en este tema fueron del tipo transversal y de corta duración, mostrando un impacto negativo en pacientes celíacos. En un estudio de desafío abierto realizado por Chartrand, se encontró que los productos "libres de gluten" que contenían almidón de trigo causaban síntomas abdominales en algunos pacientes celíacos ${ }^{34}$. Sin embargo, en otro estudio, de un año de duración y realizado en pacientes adultos asignados al azar a una DLG con o sin almidón de trigo, aunque mostró que la recuperación de la mucosa no fue completa en todos los pacientes, no demostró diferencias entre los 2 grupos en la morfología de la mucosa, la densidad de linfocitos intraepiteliales, anticuerpos séricos, densidad mineral ósea y estado nutricional después de 1 de año ${ }^{35}$. Actualmente el almidón de trigo se usa en la DLG en Europa, mientras que no se recomienda en América del Norte.

Otro aspecto que comentaremos son las deficiencias nutricionales. Numerosos estudios documentan el impacto de la mala absorción de nutrientes tanto en niños como en adultos, incluso cuando no es evidente un síndrome de malabsorción ${ }^{36}$. Un estudio realizado por Bona mostró que la baja ingesta alimentaria o la mala absorción de vitaminas del complejo B, hierro y ácido fólico parecen parcialmente responsables del retraso de la pubertad en niños con $\mathrm{EC}^{37}$. En adultos, también se ha descrito correlación entre la deficiencia de zinc y severidad del aplanamiento de las vellosidades, junto con lesiones histológicas más severas en sujetos cuyos niveles de hierro, cobre, ácido fólico y vitamina B-12 eran menores ${ }^{38}$. La ingesta de calcio, vitamina $\mathrm{D}$, magnesio y fibra (especialmente la fibra soluble), están limitados en la DLG. Es importante destacar que en Chile los productos "libres de gluten" no están fortificados, lo que aumenta la posibilidad de deficiencias de nutrientes. Los productos "libres de gluten" en general, no sólo carecen de las fortificaciones más habituales, también resultan más bajos en fibra, hierro, ácido fólico, tiamina, riboflavina y niacina ${ }^{39,40}$.

Un último tema que queremos mencionar es la malnutrición por exceso u obesidad, cada vez más frecuente como presentación inicial de la enfermedad celíaca. Un estudio de cohorte retrospectivo de 679 pacientes con enfermedad celíaca confirmada por biopsia, que se centró en las modificaciones del peso corporal en sujetos siguiendo una DLG, mostró que el IMC promedio fue de 24 , con un bajo número de sujetos con sobrepeso y obesidad. Después de estar en una DLG de 39,5 meses (en promedio), el IMC promedio aumentó a 24,6, consiguiendo que la mayoría de los pacientes lograran un peso normal; aquellos pacientes con sobrepeso y obesidad permanecieron sin 
cambios $^{41}$. En la práctica diaria, el riesgo de aumento de peso es frecuente, considerando que los pacientes al iniciar la DLG se sienten mejor, su intestino mejora y aprenden a comer productos sin gluten sin sentir molestias. Esta es una de la razones de porqué es importante que el paciente celíaco en su seguimiento sea manejado por el equipo de salud, incluyendo no sólo al médico (gastroenterólogo y de atención primaria) sino también al nutricionista. La consejería acerca del control del peso debe ser parte de los controles nutricionales periódicos. La DLG puede ser una dieta saludable, pero frecuentemente es baja en fibra, alta en grasas y deficiente en vitaminas del complejo $\mathrm{B}$; todo esto como resultado de la falta de la fortificación de las harinas utilizadas ${ }^{42}$ y el añadido de grasa para mejorar los productos elaborados sin gluten. Hoy día en que la obesidad es la gran epidemia mundial, es responsabilidad del equipo de salud el mantener la dieta del celíaco con características saludables. Una estrategia que ha tenido buenos resultados es la de incorporar productos que contengan granos alternativos, que no contienen gluten ${ }^{43}$.

En resumen, la EC ha demostrado tener una prevalencia alta y presentaciones clínicas diversas. El acceso a anticuerpos específicos de la enfermedad y el concepto de "búsqueda activa" han mejorado sustancialmente la capacidad diagnóstica, aunque hay que tener presente que si no se conocen los niveles de IgA del paciente los exámenes habituales (IgA-ENMA e IgA-tTG2) pueden dar en falsos negativos. De su patogenia se sabe que existen factores genéticos (presencia de HLA-DQ2/DQ8) que dan la susceptibilidad, pero no explican la enfermedad en su totalidad. El diagnóstico -salvo excepción- continúa basándose en la biopsia de intestino delgado. La biopsia debe hacerse siempre antes de iniciar la dieta sin gluten, mientras el paciente tiene una ingesta habitual de gluten. La enfermedad tiene tratamiento efectivo mediante la "dieta libre de gluten", que debe ser permanente, estricta y por toda la vida. El paciente debe ser seguido periódicamente por el equipo de salud, especialmente médico y nutricionista, que deben mantenerlo informado de cómo llevar a cabo los controles periódicos y las indicaciones alimentarias, promoviendo -como en toda la población- una alimentación saludable que lo proteja de la obesidad.

Potenciales conflictos de interés: Este trabajo cumple con los requisitos sobre consentimiento/asentimiento informado, comité de ética, financiamiento, estudios animales y sobre la ausencia de conflictos de intereses según corresponda.

Financiamiento: sin financiamiento.

\section{Referencias}

1.- Kang JY, et al: Systematic review: worldwide variation in the frequency of coeliac disease and changes over time. Aliment Pharmacol Ther 2013; 38 (3): 226-45.

2.- Corrao $G$, et al: Mortality in patients with coeliac disease and their relatives: a cohort study. Lancet 2001; 358 (9279): 356-61.

3.- Peters $U$, et al: Causes of death in patients with celiac disease in a population-based Swedish cohort. Arch Intern Med 2003; 163 (13): 1566-72.

4.- Rubio-Tapia A, et al: ACG clinical guidelines: diagnosis and management of celiac disease. Am J Gastroenterol 2013; 108 (5): 656-76; quiz 677.

5.- Husby $S$, et al: European Society for Pediatric Gastroenterology, Hepatology, and Nutrition guidelines for the diagnosis of coeliac disease. J Pediatr Gastroenterol Nutr 2012; 54 (1): 136-60.

6.- Catassi $C$, et al: The coeliac iceberg in Italy. A multicentre antigliadin antibodies screening for coeliac disease in school-age subjects. Acta Paediatr Suppl 1996; 412: 29-35.

7.- Hawkes ND, et al: Incidence and presentation of coeliac disease in South Glamorgan. Eur J Gastroenterol Hepatol 2000; 12 (3): 345-9.

8.- Kupfer SS, Jabri B: Pathophysiology of celiac disease. Gastrointest Endosc Clin N Am 2012: 22 (4): 639-60.

9.- Marsh MN: Gluten, major histocompatibility complex, and the small intestine. A molecular and immunobiologic approach to the spectrum of gluten sensitivity ('celiac sprue'). Gastroenterology 1992; 102 (1): 330-54.

10.- Sapone A, et al: Spectrum of gluten-related disorders: consensus on new nomenclature and classification. BMC Med 2012; 10: 13.

11.- Mustalahti $K$, et al: The prevalence of celiac disease in Europe: results of a centralized, international mass screening project. Ann Med 2010; 42 (8): 587-95.

12.- Fasano A, et al: Prevalence of celiac disease in at-risk and not-at-risk groups in the United States: a large multicenter study. Arch Intern Med 2003; 163 (3): 286-92. 
13.- Whyte LA, Jenkins HR: The epidemiology of coeliac disease in South Wales: a 28-year perspective. Arch Dis Child 2013; 98 (6): 405-7.

14.- Catassi $C$, et al: Coeliac disease in the year 2000: exploring the iceberg. Lancet 1994; 343 (8891): 200-3.

15.- Collin P: Should adults be screened for celiac disease? What are the benefits and harms of screening? Gastroenterology 2005; 128 (4 Suppl 1): S104-8.

16.- Ministerio de Salud (Minsal) Chile, Encuesta Nacional de Salud (ENS) Chile 2009-2010. 2010: Santiago.

17.- Araya M, et al: Celiac disease in a Chilean population carrying Amerindian traits. J Pediatr Gastroenterol Nutr 2000; 31 (4): 381-6.

18.- Admou B, et al: Atypical celiac disease: from recognizing to managing. Gastroenterol Res Pract 2012; 637187.

19.- Sollid LM, et al: Evidence for a primary association of celiac disease to a particular HLA-DQ alpha/beta heterodimer. J Exp Med 1989; 169 (1): 345-50.

20.- Fasano A, et al: Federation of International Societies of Pediatric Gastroenterology, Hepatology, and Nutrition consensus report on celiac disease. J Pediatr Gastroenterol Nutr 2008; 47 (2): 214-9.

21.- Anderson RP, et al: In vivo antigen challenge in celiac disease identifies a single transglutaminase-modified peptide as the dominant A-gliadin T-cell epitope. Nat Med 2000; 6 (3): 337-42.

22.- Maiuri L, et al: Association between innate response to gliadin and activation of pathogenic $\mathrm{T}$ cells in coeliac disease. Lancet 2003; 362 (9377): 30-7.

23.- See J, Murray JA: Gluten-free diet: the medical and nutrition management of celiac disease. Nutr Clin Pract 2006; 21 (1): 1-15.

24.- Silano M, Agostoni C, Guandalini S: Effect of the timing of gluten introduction on the development of celiac disease. World J Gastroenterol 2010; 16 (16): 1939-42.

25.- Agostoni $C$, et al: Complementary feeding: a commentary by the ESPGHAN Committee on Nutrition. J Pediatr Gastroenterol Nutr 2008; 46 (1): 99-110.

26.- Janatuinen $E K$, et al: No harm from five year ingestion of oats in coeliac disease. Gut 2002; 50 (3): 332-5.

27.- Peraaho $M$, et al: Effect of an oats-containing glutenfree diet on symptoms and quality of life in coeliac disease. A randomized study. Scand J Gastroenterol 2004; 39 (1): 27-31.

28.- Reunala $T$, et al: Tolerance to oats in dermatitis herpe- tiformis. Gut 1998; 43 (4): 490-3.

29.- Hoffenberg EJ, et al: A trial of oats in children with newly diagnosed celiac disease. J Pediatr 2000; 137 (3): 361-6.

30.- Hogberg L, et al: Oats to children with newly diagnosed coeliac disease: a randomised double blind study. Gut 2004; 53 (5): 649-54.

31.- Storsrud S, Hulthen LR, Lenner RA: Beneficial effects of oats in the gluten-free diet of adults with special reference to nutrient status, symptoms and subjective experiences. Br J Nutr 2003; 90 (1): 101-7.

32.- Lundin $K E$, et al: Oats induced villous atrophy in coeliac disease. Gut 2003: 52 (11): 1649-52.

33.- Arentz-Hansen $H$, et al: The molecular basis for oat intolerance in patients with celiac disease. PLoS Med 2004; 1 (1): e1.

34.- Chartrand LJ, et al: Wheat starch intolerance in patients with celiac disease. J Am Diet Assoc 1997; 97 (6): 6128.

35.- Peraaho $M$, et al: Wheat-starch-based gluten-free products in the treatment of newly detected coeliac disease: prospective and randomized study. Aliment Pharmacol Ther 2003; 17(4): 587-94.

36.- Botero-López JE, et al: Micronutrient deficiencies in patients with typical and atypical celiac disease. J Pediatr Gastroenterol Nutr 2011; 53 (3): 265-70.

37.- Bona G, Marinello D, Oderda G: Mechanisms of abnormal puberty in coeliac disease. Horm Res 2002: 57 Suppl 2: 63-5.

38.- Jameson $S$ : Coeliac disease, insulin-like growth factor, bone mineral density, and zinc. Scand J Gastroenterol 2000; 35 (8): 894-6.

39.- Thompson T: Thiamin, riboflavin, and niacin contents of the gluten-free diet: is there cause for concern? J Am Diet Assoc 1999; 99 (7): 858-62.

40.- Thompson T: Folate, iron, and dietary fiber contents of the gluten-free diet. J Am Diet Assoc 2000; 100 (11): 1389-96.

41.- Kabbani TA, et al: Body mass index and the risk of obesity in coeliac disease treated with the gluten-free diet. Aliment Pharmacol Ther 2012; 35 (6): 723-9.

42.- Hallert $C$, et al: Evidence of poor vitamin status in coeliac patients on a gluten-free diet for 10 years. Aliment Pharmacol Ther 2002; 16 (7): 1333-9.

43.- Lee AR, et al: The effect of substituting alternative grains in the diet on the nutritional profile of the gluten-free diet. J Hum Nutr Diet 2009; 22 (4): 359-63. 